UCRL-JC-121694

PREPRINT

\title{
Adiabatic Compression of a Closed-Field-Line Configuration by a Centimeter-Size Liner
}

\author{
R. P. Drake, J. H. Hammer, C. W. Hartman, L. J. Perkins, and D. D. Ryutov
}

This paper was prepared for submittal to the 16th Symposium on Fusion Engineering (SOFE '95)

Champaign, Illinois

September 30 - October 5, 1995

September 1995

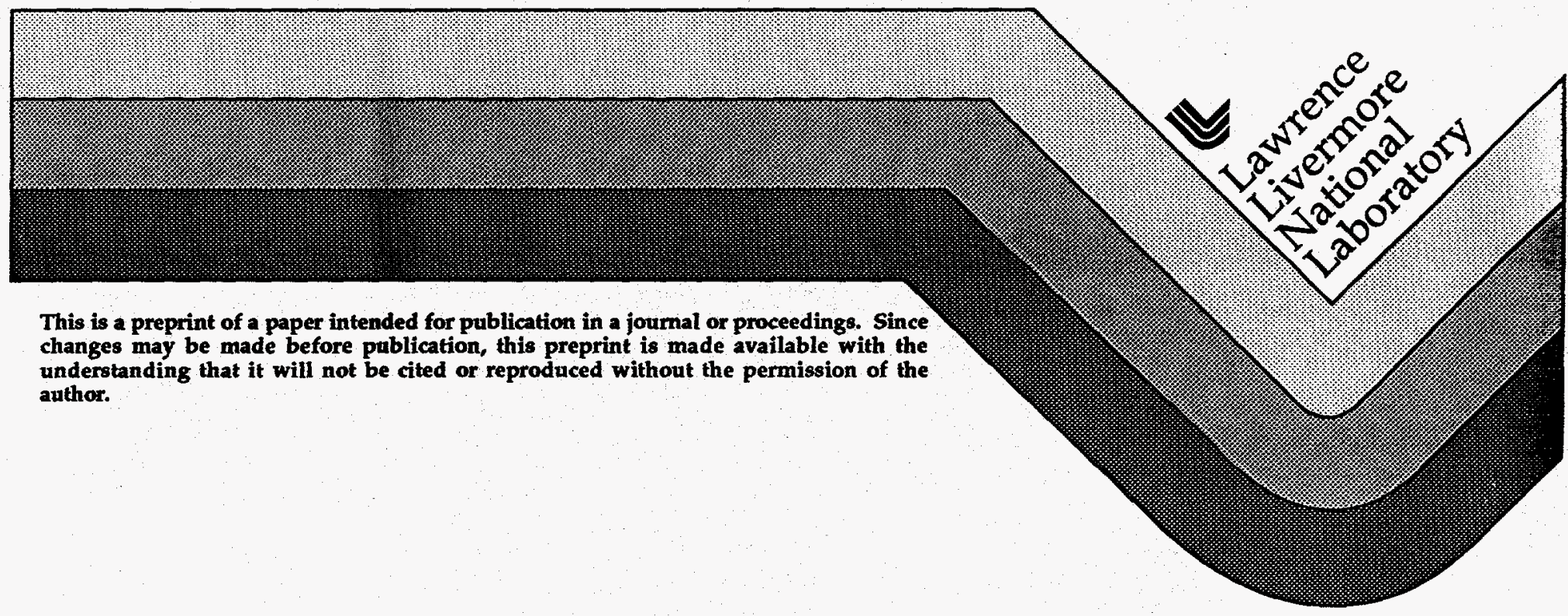


This document was prepared as an account of work sponsored by an agency of the United States Government. Neither the United States Government nor the University of California nor any of their employees, makes any warranty, express or implied, or assumes any legal liability or responsibility for the accuracy, completeness, or usefulness of any information, apparatus, product, or process disclosed, or represents that its use would not infringe privately owned rights. Reference herein to any specific commercial product, process, or service by trade name, trademark, manufacturer, or otherwise, does not necessarily constitute or imply its endorsement, recommendation, or favoring by the United States Government or the University of California. The views and opinions of authors expressed herein do not necessarily state or reflect those of the United States Government or the University of California, and shall not be used for advertising or product endorsement purposes.

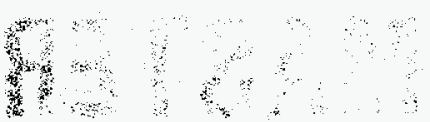




\title{
Adiabatic Compression of a Closed-Field-Line Configuration by a Centimeter-Size Liner ${ }^{+}$
}

\author{
R.P.Drake, J.H.Hammer, C.W.Hartman, L.J.Perkins, and D.D.Ryutov ${ }^{++}$ \\ Lawrence Livermore National Laboratory, Livermore, CA 94550, USA
}

\begin{abstract}
The paper considers adiabatic compression of a pre-formed closed field line configuration by an imploding liner. Three configurations are discussed: the field-reversed configuration, the spheromak and the Z-pinch. It is shown that, by employing a 2D-compression, one can reach a break-even condition with an energy input as low as $100 \mathrm{~kJ}$. It is emphasized that the possibility of performing crucial experiments on small-tomedium-scale experimental devices may considerably shorten the development path for the system under consideration.
\end{abstract}

\section{INTRODUCTION}

Imploding liners have been considered as a way for generating fusion-grade plasma since the '70s. However, in these early assessments, systems with large dimensions and, accordingly, large energy inputs (in the range of tens and even hundreds MJ) were considered. During the last decade, remarkable progress has been made in two areas of plasma technology and plasma physics relevant to this fusion concept: i) high convergence ratios have been obtained in implosions of centimeter-size liners [1]; ii) much better understanding of the properties of dense wall-confined plasmas has been reached (see, e.g., survey [2]). Based on these achievements, we reconsidered the fusion prospects of the liner concept.

We have in in mind small initial dimensions and relatively short implosion times $\sim 1-2 \mu \mathrm{s}$. Initial liner length, radius and weight are assumed to be in the range $5-10 \mathrm{~cm}, 1-2 \mathrm{~cm}$, and $5-10 \mathrm{~g}$, respectively. We consider an option provided by a self-similar implosion of the liner in both $\mathrm{r}$ - and $\mathrm{z}$-directions and show that this mode of implosion has advantages over purely radial implosion (in what follows, we call this $r-z$ mode a $2 D$ implosion; we call a purely radial mode a $1 \mathrm{D}$ implosion). We consider only closed field line configurations which would provide good plasma thermal insulation from the liner and the end walls; we try to find conditions under which the fusion alpha particles would also be confined. We concentrate on the situation in which the initial plasma (which is to be adiabatically compressed by the imploding liner) has a pressure comparable with or exceeding the initial magnetic pressure of the magnetic field inside the liner, so that essentially all the energy of the implosion is spent on the plasma heating and not on the increase of the energy of the magnetic field immersed into the plasma. What we find is that, with all these ingredients combined together, it is probably possible to achieve a plasma density $\sim 10^{21} \mathrm{~cm}^{-3}$ and fusion break-even $(Q=1)$ at a plasma energy level $\sim 0.1 \mathrm{MJ}$ while values of $Q$ as high as 5-6 are probably within the reach of the experiments with a plasma energy content $\sim 1 \mathrm{MJ}$. The present paper is based on our recent report [3] which contains a more detailed analysis of various physics and engineering issues of liner implosions.

In the same range of input energies, another compact pinch system may work: this is a so-called staged Z-pinch ( see recent paper [4] and references therein). A staged Z-pinch is supposed to use considerably lighter (e.g., gaseous) liners and to obtain much higher final densities $\left(-10^{24} \mathrm{~cm}^{-3}\right)$. The physics involved may be considerably different but the required experimental hardware is very similar, so that two sets of liner experiments can be, in principle, carried out on the same device.

\section{GENERAL DESCRIPTION OF THE CONCEPT}

A possible geometry of the system is illustrated by Fig.1. This figure depicts a situation when the magnetic configuration is of the FRC type, with the field reversal maintained by plasma currents (not by an electron or ion beam). Other possible configurations will be discussed later.

We characterize the initial state of the FRC by its initial beta value,

$$
\beta_{0}=8 \pi \mathrm{p} 0 / \mathrm{B}_{0}^{2}
$$

Here and below, the subscript " 0 " denotes the initial values of the corresponding quantities. As we are going to restrict ourselves only to order-of-magnitude estimates, we don't precisely specify the point in which $\mathrm{p}_{0}$ and $\mathrm{B}_{0}$ entering (1) are taken: this is just some "characteristic" point inside the FRC.

As is well known, initially a magnetically confined FRC has $\boldsymbol{\beta}_{0} \sim 1$ as a "natural" state (see, e.g., survey [5]). For the system under consideration, with the confinement volume restricted on all sides by conducting material walls, an FRC configuration with $\beta_{0}>1$ is also possible. Therefore, we consider $\beta_{0}$ as a free parameter that is a subject to constraint

$$
\beta_{0} \geq 1
$$

\footnotetext{
+ This work was carried out under the auspices of the U.S. Department of Energy by Lawrence Livermore National Laboratory under Contract W-7405-Eng-48

${ }^{++}$On leave from Budker Institute of Nuclear Physics, Novosibirsk 630090, Russia
} 
After the initial configuration is prepared, an external voltage is applied between the electrodes 2 and 3 (Fig.1) with a pulselength short compared to the liner skin-time. The axial current is then excited only in the liner and does not penetrate into the plasma volume. The current must be large enough that the pressure of the azimuthal $(\varphi)$ component of the driven magnetic field considerably (at least by a factor of a few) exceeds the initial plasma pressure inside the liner. Then the liner gets accelerated in the inward direction and stores considerable kinetic energy. After the liner radius decreases several times with respect to its initial value, the plasma pressure inside the liner becomes larger than the magnetic pressure outside the liner, and the liner decelerates. At the point of maximum compression, it stops and then expands again under the action of the plasma pressure.

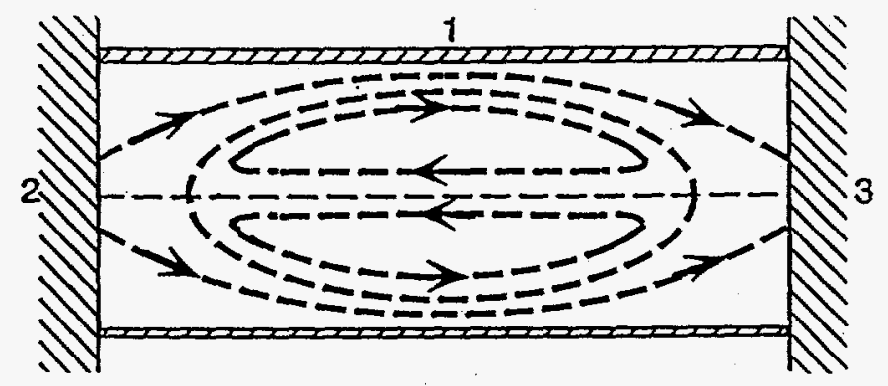

Fig.1. A schematic showing the geometry of the implodingliner system.The liner (1) will implode to adiabatically compress the plasma and the closed magnetic-field configuration. Our reference case of an FRC is shown by the dashed lines. The structures (2) and (3) are the electrodes which drive the current that implodes the liner.

The liner is assumed to be made of some heavy material and its velocity during all phases of the implosion is assumed to be much smaller than the plasma sound velocity. Therefore, plasma compression occurs in the adiabatic manner.

Of most interest is the dynamics of the system near the point of the maximum compression where the fusion "flash" can occur. For the plasma parameters that are typical for our system, the maximum plasma pressure is such that the compressibility of the liner material becomes important. In order to eliminate the adverse effect of the liner compressibility (which reduces possible compression rates), we consider only thin liners, whose thickness even in the final state is less than the plasma radius in this state. In such a case, a first rough assessment of the liner dynamics can be carried out under the approximation that the liner is just an infinitely thin massive surface; the liner compressibility for a thin liner becomes, obviously, unimportant. This model of a structureless liner will be used throughout this paper. The liner will be characterized by its linear mass density $\mu$ (mass per unit length in the $\mathrm{z}$-direction).

We introduce the elongation of the FRC as the ratio of its length $\mathrm{L}$ to its diameter $2 \mathrm{a}$,

\section{$: \quad E=\mathrm{L} / 2 \mathrm{a}$}

1

We consider a self-similar $2 \mathrm{D}$ compression which maintains a constant value of $E$. This means that the FRC should be compressed not only radially but also axially. A concrete way of achieving this mode of compression is to properly tailor the mass density of the liner along the axis [5]: if the central part is heavier than the ends, its compression lags behind, maintaining an approximate constancy of $E$ of the plasma object nesting inside the liner. Exact constancy of $E$ is not of principal importance. However, since current experiments with FRC's show the best performance for $E=3-4$, we believe that it would be reasonable to maintain $E$ relatively close to $3-4$. Another reason for choosing $E=3-4$ is that this value seems convenient from the viewpoint of the implosion physics: (not too large to make the kink instabilities of a great concern and not too small to make the electrode effects dominant).

Other possible candidates for configurations to be placed into the liner are the prolate spheromak and the Z-pinch configuration. In this latter case the azimuthal component of the magnetic field inside the liner does not necessarily serve for the plasma confinement (which can be provided by the material walls), and the Bennett relationship does not necessarily hold. In terms of the problem under consideration, the spheromak configuration does not differ much from the FRC. If the prolate spheromak can be MHD stable at $\beta>1$, it should be considered as a good candidate for the adiabatic compression experiment. An obvious difference of the Z-pinch configuration from the other two is that in the Z-pinch geometry the particle drift trajectories intersect the walls (more specifically, the end walls). Still, for low-Q systems this may not be too a severe problem. As for the drift losses of the alpha particles in the Z-pinch, they occur within such a short time that one can't count on any alpha-particle heating. This is of little concern for the low-Q systems but may become important at $Q>5$.

The obvious advantage of the Z-pinch for "filling" a liner is that this configuration can be easily created inside the liner, just by using a simple electrode discharge. The preparation of a miniature FRC or a spheromak will probably require some ingenuity. One possible approach could be the preparation of these objects outside the liner and translation of them into the liner through a hole in one of the electrodes. Then, the liner implosion should be organized in such a way that, on the hole side of the discharge, the liner would collapse on the axis relatively early in time, to prevent the ejection of the plasma back into the preparation chamber. The preparation of the FRC and spheromak inside the liner is also conceivable. In this case, the liner would have to have cuts in the axial direction, to allow the $\mathrm{Z}$-component of the magnetic field to penetrate into the liner. In case of a spheromak, one can in principle count on the helicity injection technique.

It's quite clear that, under the action of the heat flow from the plasma and Joule dissipation in the skin-layer at the inner side of the liner, this inner surface will be evaporated and ionized. As 
both the ion gyroradius and mean free path of these relatively cold ions will be 2-3 orders of magnitude smaller than the plasma radius, they will not directly penetrate into the plasma. In addition, it turns out that, in a high beta plasma, the impurities get repelled from the hot region, under the action of the thermal force and plasma convection towards the walls.

\section{PARAMETERS OF A CONCEIVABLE EXPERIMENT}

Analysis presented in Ref.[3] has shown that even with quite pessimistic assumptions regarding plasma thermal conductivity to the liner walls, thermal losses do not constitute a factor that limits the quality of pellet performance. Bremsstrahlung also constitutes an insignificant energy sink (unless one deliberately seeds the plasma with heavy impurities, in onder to use the system under consideration as a pulsed source of X-rays).

Table 1 presents possible set of plasma parameters in the experiment where energy deposited to the plasma was $100 \mathrm{~kJ}$, with total neglect of energy losses from the plasma.

Table 1.

Parameters of a Conceivable Experiment

\begin{tabular}{lcc}
\hline \hline Parameter & Initial state & Final state \\
\hline \hline Plasma temperature, $\mathrm{keV}$ & 0.1 & 10 \\
Magnetic field, $\mathrm{T}$ & 10 & $10^{3}$ \\
Plasma beta & .1 & 10 \\
Plasma density, cm ${ }^{-3}$ & $1.25 \cdot 10^{18}$ & $1.25 \cdot 10^{21}$ \\
Plasma radius, cm & 1 & 0.1 \\
Plasma elongation, $E$ & 3 & 3 \\
Plasma energy, $\mathrm{kJ}$ & 1 & 100 \\
Magnetiz. parameter $\omega_{\mathrm{Bi}} \tau_{\mathrm{i}}$ & 1 & 100 \\
Parameter s=a/ $\rho_{\mathrm{i}}$ & 40 & 40 \\
Collisionality $\mathrm{a} / \lambda_{\mathrm{ii}}$ & 40 & 0.4
\end{tabular}

For this set of parameters, the gyroradius of fusion alphaparticles in the final state is equal $0.03 \mathrm{~cm}$ and is smaller by a factor of 3 than a plasma radius. Therefore, if the drift trajectories of the alpha particles are closed inside the plasma volume (as in the FRC and Spheromak configurations), a considerable fraction of the alphas is confined and deposits its energy in the plasma. In the Z-pinch geometry, the alphas will drift away along the axis before depositing any substantial amount of their energy into the plasma.

In the Table 2 we present a possible set of the parameters of the liner and the value of the initial external current that are compatible with the plasma parameters listed in Table 1 . We show the liner parameters in the equatorial plane. We choose the initial liner thickness in a such a way that the final liner thickness is equal to a half of plasma diameter in the final state.

Table 2

Parameters of the Compressing System

$\begin{array}{ll}\text { Initial liner radius, } \mathrm{cm} & 1 \\ \text { Final liner radius, cm } & 0.1 \\ \text { Initial liner thickness, cm } & 0.01 \\ \text { Final liner thickness, cm } & 0.1 \\ \begin{array}{l}\text { Current through the liner, MA } \\ \text { Initial magnetic field at the }\end{array} & 5 \\ \text { outer surface of the liner, } \mathrm{T} & 100 \\ \text { Liner density, } \mathrm{g} / \mathrm{cm}^{3} & 20 \\ \text { Total compression time, } \mu \mathrm{s} & 2\end{array}$

Liner dwell time (Eq. (39) near the point of maximum compression, $\mu$ s 0.15

In our considerations we have assumed that the Z-current that drives the liner does not penetrate through the liner into the compressed plasma. This is certainly true if the liner thickness exceeds the skin-depth for the characteristic time of order of $\tau_{\text {total }}$. For the typical set of parameters listed in Tables 1 and 2, $\tau_{\text {total }}$ exceeds the current penetration time by a factor of $2-3$, so that some of the driving current would switch to the plasma. However, until very late in the compression phase the plasma conductivity is orders of magnitude smaller than the liner conductivity and the fraction of the driving current switched to the plasma remains small.

If necessary, one can completely eliminate any penetration of the driving current into the plasma by using a two-layer liner. The outer layer can be made of aluminum and have a thickness exceeding the skin-depth; the inner layer should be made of a heavy material. A possible example for the parametric domain covered by Tables 1 and 2 could be: $0.3 \mathrm{~mm}$ of $A 1 \quad p=2.7$ $\left.\mathrm{g} / \mathrm{cm}^{3}\right)$ and $0.1 \mathrm{~mm}$ of $\mathrm{Au}\left(\rho=18 \mathrm{~g} / \mathrm{cm}^{3}\right)$. The outer shell is lighter than the inner one and will not considerably affect the implosion dynamics. It will prevent the driving current from penetrating into the heavy inner shell and into the plasma. The presence of this thick outer shell will also eliminate any problems of a premature thermal explosion of the inner part of the liner.

As it turns out (see[3]) the factor that limits plasma gain Q, is a relatively short liner dwell time near the turning point. The $Q$ value determined by this process can be presented as:

$$
\mathrm{Q}=0.2\left(\beta_{0}{ }^{1 / 3} \rho_{\mathrm{L}} / E^{2 / 3} \mathrm{~T}_{0}\right)^{1 / 2}\left(\mathrm{~W}_{\max } \mathrm{B}_{0}\right)^{1 / 3}
$$

Here liner density is measured in $\mathrm{g} / \mathrm{cm}^{3}$, initial temperature $\mathrm{T}_{0}$ in $\mathrm{eV}$, plasma energy content in the final state in $\mathrm{kJ}$ and initial 
magnetic field in $T$. We remind that $\beta_{0}$ is initial plasma beta and $E$ is plasma elongation (3).

\section{REACTOR ISSUES}

We can write the net power generated by an electricityproducing plant based on our liner-driven capsule scheme as:

$$
P_{e, \text { net }}=r Q W\left(1-f_{\text {aux }}\right)\left[0.8 \mathrm{M} \eta_{\text {th }}-1 /\left(\mathrm{Q} \eta_{\mathrm{i}}\right)\right]
$$

where $r$ is the repetition rate, $W$ is the input drive energy (i.e. $Q W$ is the fusion energy released per shot), $M$ is the blanket energy multiplication, $\eta_{i}$ is the efficiency of conversion from wall plug energy to input energy, $\eta_{\text {th }}$ is the thermal cycle efficiency, and where $f_{\text {aux }}$ is the fraction of gross electrical power required to supply the plant auxiliaries other than the energy input to the fusion core itself. Equation (5) assumes that no useful electrical power is generated from either the input power $(\mathrm{rW})$ or the fusion alpha power $(\mathrm{rQW} / 5)$.

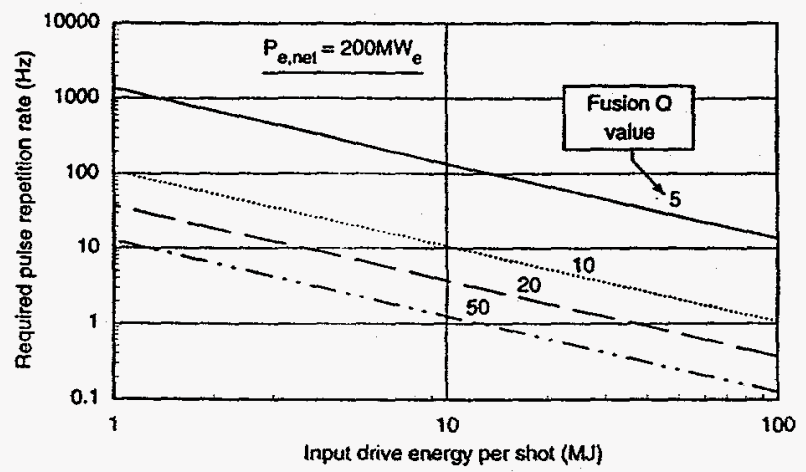

Fig 2 Energy parametrics for a $200 \mathrm{MW}$ e pulsed, liner-driven FRC reactor. The required pulse repetition frequency is plotted as a function of the input drive energy for various values of the fusion gain $Q$. Assumptions on plant performance are supplied in the text.

Taking typical values of, say, $\mathrm{M} \sim 1.3, \eta_{\mathrm{th}} \sim 0.35, \eta_{\mathrm{i}} \sim 0.6$ and $f_{\text {aux }}-0.05$, Fig 2 shows the required repetition rate $r$ to sustain a $200 \mathrm{MW}$ e,net reactor as a function of the input energy $W$ to the liner-driven capsule for various values of the fusion $Q$ value. Note that no net electric power is possible for such a reactor for $Q$ values less than about 5. Great benefits are seen by increasing the $Q$ to the range of 20 to 30 . In particular, for $Q=20$, an input energy of $12 \mathrm{MJ}$ would require a pulse repetition rate of only $-3 \mathrm{~Hz}$, surely feasible for a repetitive mechanical target system such as this. Under such conditions, the energy loading on a liquid first wall would be $300 \mathrm{MJ}$. This is very similar to that of an ICF reactor although there would be a greater momentum transfer from the liner mass relative to the hohlraum mass of an indirect-drive ICF capsule. Certainly, the $3 \mathrm{~Hz}$ repetition rate is sufficiently low to allow for adequate chamber clearing between shots. Therefore, the further work on the proposed scheme should be concentrated on the ways of a considerable increase of $Q$.
Relationship (4) shows that the plasma enhancement factor $Q$ depends relatively weakly on the parameters of the system. For example, in order to increase $Q$ from 1.5 to 3 , one would have to increase $\mathrm{W}_{\max }$ to $0.8 \mathrm{MJ}$. Therefore, in its present form, the concept we are considering, would work as the basis of an energy producing facility only at the level of the input energies exceeding $10 \mathrm{MJ}$. Potentially, Q can be increased somewhat by lengthening the liner dwell time near the point of a maximum compression. One possible way of achieving this objective, is an ablation of some amount of the liner material near the maximum compression point and using the resulting reactive force to balance the plasma pressure near the turning point. The ablation could be produced by a pulsed laser. Simple estimates show that an increase of $Q$ by a factor of 2 is possible in this way but more detailed assessment would be desirable. Another seemingly obvious way of increasing $Q$ is just increasing the liner thickness but the evaluation of the potentialities of the thicker liners would require a more detailed study.

One discouraging feature of the conventional approaches to fusion energy is that they do not appear to lend themselves to a small reactor for developmental purposes. This is in contrast with the normal evolution of a new technology which typically proceeds to a full scale commercial plant via a set of graduated steps. Therefore, the prospects for the near term realization of fusion, and perhaps the ultimate commercial reactor, would be improved, if it could be introduced through a compact, cheap system such as the wall-confined system of this paper.

\section{REFERENCES}

[1] J.H. Degnan, F.M. Lehr, J.D. Beason, G.P. Baca, D.E.Bell, A.L. Chesley, D. Dietz, D.B. Dunlap, S.E. Englert, T.J. Englert, D.G. Gale, J.D. Graham, J.J. Havranek, C.D. Holmberg, T.W. Hussey, R.A. Lewis, C.A. Outten, R.E. Peterkin,Jr., D.W. Price, N.F. Roderick, E.L. Ruden, U. Shumlak, G.A. Smith, P.J. Turchi. "Electromagnetic implosion of spherical liner", Phys. Rev. Letters, vol.74, 98101, January 1995.

[2] G.E.Vekshtein. In: Reviews of Plasma Physics (B.B.Kadomtsev, Ed), v. 15, p.1, Consultants Bureau, 1990

[3] R.P.Drake, J.H.Hammer, C.W.Hartman, L.J.Perkins, and D.D.Ryutov. "Sub-Megajoule Liner Implosion of a Closed Field Line Configuration". Sbmitted to Fusion Technology.

[4] A. Hoffman. "Reactor Prospects and Present Status of Field Reversed Configurations". Transactions of Fusion Technology, vol. 27, 91-96, April 1995.

[5] E.P. Velikhov, private communication (1971)

[6] G.E.Vekshtein, D.D.Ryutov, P.Z.Chebotaev. "Diffusion of heavy impurities in a dense, wall confined plasma”. Sov J.Plasma Phys., vol.1, 220-224, March 1975 


\section{DISCLATMER}

Portions of this document may be illegible in electronic image products. Images are produced from the best available original document. 